Pacific Journal of Mathematics

REPRESENTATION AND SERIES SUMMABILITY OF
COMPLETE BIORTHOGONAL SEQUENCES 


\title{
REPRESENTATION AND SERIES SUMMABILITY OF COMPLETE BIORTHOGONAL SEQUENCES
}

\author{
WILLIAM H. RUCKLE
}

Let $\left\{x_{j}, x_{j}^{\prime}\right\}$ be a complete biorthogonal sequence with each $x_{j}$ in a Banach space $X$ and each $x_{j}^{\prime}$ in the conjugate space $X^{*}$. A study is made of the summability properties of the series $\sum_{j} x_{j}^{\prime}(x) x_{j}$ and $\sum_{j} x_{j}^{\prime}(x) x^{\prime}\left(x_{j}\right)$ as $x$ ranges over $X$ and $x^{\prime}$ over $X^{*}$. Conditions are given for matrix and abstract methods of series summability for these series in terms of certain sequence spaces which arise naturally in connection with the biorthogonal sequence.

Fundamental work on complete biorthogonal sequences in Banach spaces was done by Banach in Chapter VII of [1] and by Frink in [3]. Further contributions were made by others including Kadets and Pełczynski in [6] where the concept of a norming biorthogonal sequence was defined. There are various types of biorthogonal sequences which generalize the Schauder basis and these are discussed in [11].

Let $\left\{x_{j}, x_{j}^{\prime}\right\}$ be a complete biorthogonal sequence with each $x_{j}$ in a Banach space $X$ and each $x_{j}^{\prime}$ in the conjugate space $X^{*}$. In $\S 3$ four spaces of scalar sequences are defined in connection with this biorthogonal sequence: (a) $S$, the associated sequence space, consisting of all sequences $\left\{x_{j}^{\prime}(x)\right\}$ as $x$ ranges over $X$; (b) $S^{f}$ which consists of all sequences $\left\{x^{\prime}\left(x_{j}\right)\right\}$ as $x^{\prime}$ ranges over $X^{*}$; (c) $M(S)$, the multiplier algebra; $\left(a_{j}\right) \in M(S)$ if and only if $\left\{a_{j} x_{j}^{\prime}(x)\right\} \in S$ whenever $x \in X$; (d) $\mathscr{S}(\mathbf{S})$, the series space, which is, roughly speaking, the smallest Banach sequence space which contains all sequences of the form $\left\{x_{j}^{\prime}(x) x^{\prime}\left(x_{j}\right)\right\}$ as $x$ ranges over $X$ and $x^{\prime}$ over $X^{*}$. Note that these sequences are the terms of the numerical expansion

$$
x^{\prime}(x) \sim \sum_{j} x_{j}^{\prime}(x) x^{\prime}\left(x_{j}\right) .
$$

The object of this paper is to represent these four sequence spaces, determine their relationship and apply them to determine properties of the biorthogonal sequence $\left\{x_{j}, x_{j}^{\prime}\right\}$ and the space $X$. Particular attention is paid to the series summability of (1.1) and

$$
x \sim \sum_{j} x_{j}^{\prime}(x) x_{j}
$$

The most important results are Theorem 6.4 where conditions are noted under which there is a continuous linear functional on $\mathscr{S}(S)$ which gives to each series $\left\{x_{j}^{\prime}(x) x^{\prime}\left(x_{j}\right)\right\}$ its "correct" sum and Theorem 
7.2 where conditions are given for the existence of a row finite matrix with $u_{n}(j)$ in the $n$th row $j$ th column such that

$$
x=\lim _{n} \sum_{j} u_{n}(j) x_{j}^{\prime}(x) x_{j}
$$

for each $x$ in $X$.

In $\S 2$ it is noted that a norming complete biorthogonal sequence can always be constructed in a separable Banach space. This strengthens a result of Markushevitch [7]. In $\S 4$ we derive necessary machinery for what follows and discuss consistency for a subset of $\varphi$.

Means of constructing all types of spaces $S$ and $S^{f}$ are given in $\S 5$. Since $S^{f}$ is isomorphic to $X^{*}$ and every separable Banach space admits a complete biorthogonal sequence, 5.4 is a representation for the dual of every separable Banach space. Also conditions are given which differentiate between norming and nonnorming biorthogonal sequences in terms of $S^{f}$.

The series space is studied in $\S 6$ and the multiplier algebra in $\S 7$. For instance, it is noted in 6.2 that $\mathscr{S}(S)$ consists of the diagonals of nuclear operators in $X$ with respect to the biorthogonal sequence $\left\{x_{j}, x_{j}^{\prime}\right\}$. Proposition 7.1 (a) generalizes Theorem 4.2 of [8] where multiplier algebras are also studied. Finally these final two sections contain the summability theorems as noted above.

2. Complete biorthogonal sequences. A double sequence $\left\{x_{j}, x_{j}^{\prime}\right\}$ with each $x$ in a linear topological space $X$ and each $x_{j}^{\prime}$ in the conjugate space $X^{*}$ is called a complete biorthogonal sequence ("in $X$ ") if

(a) For each $i$ and each $j, x_{j}\left(x_{k}\right)=\delta_{j k}$ (Kronecker $\delta$ );

(b) $\left\{x_{j}^{\prime}\right\}$ is total on $X$, i.e., $x_{j}^{\prime}(x)=0$ for $x$ in $X$ only if $x=0$;

(c) $\left\{x_{j}\right\}$ is total on $X^{*}$, i.e., $x^{\prime}\left(x_{j}\right)=0$ for $x^{\prime}$ in $X^{*}$ only when $x^{\prime}=0$.

This terminology differs somewhat from that used by Banach in note $\S 1$ to Chapter VII of [1] in that he uses a pair of sequences. $\left\{x_{j}\right\},\left\{x_{j}^{\prime}\right\}$ instead of a double sequence. A biorthogonal sequence $\left\{x_{j}, x_{j}^{\prime}\right\}$ in a Banach space $X$ is called norming if there is a subset $A$ of $\left[\left\{x_{j}\right\}\right]$, the span of $\left\{x_{j}\right\}$, such that the norm

$$
\|x\|_{A}=\sup \left\{\left|x^{\prime}(x)\right|: x^{\prime} \in A\right\}
$$

determines the topology of $X$.

THEOREM 2.1. There is a complete norming biorthogonal sequence in every separable Banach space.

Proof. Let $\left\{y_{n}\right\}$ be a sequence dense in $X$. By the Hahn-Banach theorem there is a sequence $\left\{y_{n}^{\prime}\right\}$ in $X^{*}$ such that $y_{n}^{\prime}\left(y_{n}\right)=\left\|y_{n}\right\|$ anb $\left\|y_{n}^{\prime}\right\|=1$ for each $n$. 
Let $x \in X$ and $\varepsilon>0$ be given. There is $y_{n}$ such that $\left\|x-y_{n}\right\|<\varepsilon / 2$. Then

$$
\begin{aligned}
0 & \leqq\|x\|-\left|y_{n}^{\prime}(x)\right| \leqq\left|\|x\|-y_{n}^{\prime}(x)\right| \\
& \leqq\left|\|x\|-\left\|y_{n}\right\|\right|+\left|\left\|y_{n}\right\|-y_{n}^{\prime}(x)\right| \\
& \leqq\left\|x-y_{n}\right\|+\left\|y_{n}^{\prime}\right\|\left\|y_{n}-x\right\|<\varepsilon
\end{aligned}
$$

which implies that

$$
\|x\|=\sup _{n}\left|y_{n}^{\prime}(x)\right| \text {. }
$$

Since $\left\{y_{n}\right\}$ being dense in $X$ is total on $X^{*}$ and $\left\{y_{n}^{\prime}\right\}$ is total on $X$ there is by Theorem 4 of [4] a complete biorthogonal sequence $\left\{x_{n}, x_{n}^{\prime}\right\}$ in $X$ such that $\left[\left\{x_{n}\right\}\right]=\left[\left\{y_{n}\right\}\right]$ and $\left[\left\{x_{n}^{\prime}\right\}\right]=\left[\left\{y_{n}^{\prime}\right\}\right]$. If $A$ consists of all $x^{\prime} \in X^{*}$ such that $\left\|x^{\prime}\right\| \leqq 1$ then $\left\{y_{n}^{\prime}\right\} \subset A$ so that

$$
\|x\|_{A}=\|x\|
$$

for each $x \in X$. Therefore, $\left\{x_{n}, x_{n}^{\prime}\right\}$ is norming.

The first part of the preceeding argument is frequently encountered; see, for instance, Lemma 4 of [6].

3. Sequence spaces associated with a complete biorthogonal sequence. In the following, the letters $s, t, u, v$ will always denote sequences of scalars. If $s$ is the sequence $\left\{a_{1}, a_{2}, \cdots\right\}, s(j)$ denotes the $j$ th coordinate $a_{j}$. Addition, scalar multiplication and product of sequences is defined coordinatewise. The sequence each of whose coordinates equals one is denoted by $e$; the sequence $\left\{\delta_{n j}: j=1,2, \cdots\right\}$ by $e_{n}$. A linear space of sequences on which there is a locally convex topology is called a $K$-space if each coordinate functional given by

$$
E_{j}(s)=s(j)
$$

is continuous. A $K$-space which is a Banach space is called a $B K$-space.

For $\left\{x_{j}, x_{j}^{\prime}\right\}$ a complete biorthogonal sequence in a Banach space $X$ the associated sequence space written $S\left\{x_{j}, x_{j}^{\prime}\right\}$ or simply $S$ consists of all sequences

$$
s_{x}=\left\{x_{j}^{\prime}(x): j=1,2, \cdots\right\}
$$

as $x$ range over $X$. The correspondence of $x$ in $X$ to $s_{x}$ is called the canonical isomorphism of $X$ onto $S$. If $S$ is given the topology of identification, i.e., the norm

$$
\left\|s_{x}\right\|=\|x\|
$$

then $S$ becomes a $B K$-space. The canonical isomorphism is one to one since $\left\{x_{j}^{\prime}\right\}$ is total on $X$. Under the canonical isomorphism $x_{j}$ cor- 
responds to $e_{j}$ and $E_{j}$ corresponds to $x_{j}^{\prime}$ in the sense that

$$
E_{j}\left(s_{x}\right)=x_{j}^{\prime}(x) \text {. }
$$

The dual associated sequence space written $S^{f}\left\{x_{j}, x_{j}^{\prime}\right\}$ or simply $S^{f}$ consists of all sequences

$$
t_{y}=\left\{y\left(x_{j}\right): j=1,2, \cdots\right\}
$$

as $y$ ranges over $X^{*}$ or equivalently of all sequences

$$
t_{y}=\left\{y\left(e_{j}\right): j=1,2, \cdots\right\}
$$

as $y$ ranges over $S^{*}$. With the norm

$$
\left\|t_{y}\right\|_{f}=\|y\|
$$

$S^{f}$ is isometric to $X^{*}$ (or $S^{*}$ ) under the canonical isomorphism of $y$ to $t_{y}$ since $\left\{x_{j}\right\}$ is total on $X^{*}$.

The multiplier algebra denoted by $M\left(\left\{x_{j}, x_{j}^{\prime}\right\}\right)$ or more usually by $M(S)$ consists of all sequences $u$ such that

$$
u s \in S \text { whenever } s \in S \text {. }
$$

According to 3.3 of $[8], M(S)$ with the norm

$$
\|u\|_{M}=\sup \{\|u s\|:\|s\| \leqq 1\}
$$

is a $B K$-algebra isometric to the subalgebra $\mathscr{D}$ of continuous operators $F$ from $X$ into $X$ such that

$$
x_{j}^{\prime}\left(F x_{k}\right)=0
$$

for $j \neq k$.

The series space of $\left\{x_{j}, x_{j}^{\prime}\right\}$ denoted by $\mathscr{S}(S)$ consists of all sequences $v$ having the form

$$
v=\sum_{n=1}^{\infty} s_{n} t_{n}
$$

such that each $s_{n}$ in $\varphi$, each $t_{n}$ is in $S^{f}$ and

$$
\sum_{n=1}^{\infty}\left\|s_{n}\right\|\left\|t_{n}\right\|_{f}<\infty \text {. }
$$

The infinite series in (3.1) converges coordinatewise since

$$
\sum_{n=1}^{\infty}\left|s_{n}(j)\right|\left|t_{n}(j)\right| \leqq\left\|e_{j}\right\|\left\|e_{j}\right\|_{f} \sum_{n=1}^{\infty}\left\|s_{n}\right\|\left\|t_{n}\right\|_{f} .
$$

Throughout this paper the familiar properties of $B K$-space discussed in 11.3 and 12.4 of [12] are used without citation. 
4. Absolutely convex subsets and $B K$-spaces. The space of all sequences which are eventually 0 is denoted by $\varphi$. The space of all sequences is denoted by $\omega$. The space $\omega$ given the Tychonoff topology as a countable product of scalar fields has many familiar properties which are assumed in the sequel. For $A$ a subset of $\varphi$ and $S$ an arbitrary space of sequences $A^{(S)}$ is equal to the set of all $s \in S$ such that $\left|\sum_{j} s(j) t(j)\right| \leqq 1$ for each $t$ in $A$. Thus $A^{(s)}$ is the absolute polar of $A$ in $S$ when $S$ and $\varphi$ are placed in duality by means of the bilinear form

$$
(s, t)=\sum_{j} s(j) t(j) .
$$

For $B$ a subset of $\omega, B^{(\varphi)}$ denotes the absolute polar of $B$ in $\varphi$, namely the set of all $t$ in $\varphi$ such that $|(s, t)| \leqq 1$ for each $s$ in $B$.

4.1. Let $A$ be a bounded subset of $\omega$ which is balanced but not necessarily convex, and let $p$ be the Minkowski gauge of $A$, i.e.,

$$
p(s)=\inf \{a>0: s \in a A\} .
$$

(a) If $\left\{s_{n}\right\}$ is a sequence in $\omega$ such that $\sum_{n} p\left(s_{n}\right)<\infty$ then $\sum_{n}\left|s_{n}(j)\right|<\infty$ for each $j$. that

(b) Let $S(A)$ denote the collection of all sequences $s$ in $\omega$ such

$$
s=\sum_{n} s_{n}, \sum_{n} p\left(s_{n}\right)<\infty .
$$

Then $S(A)$ is a $B K$-space with the norm

$$
\|s\|^{A}=\inf \left\{\sum_{n} p\left(s_{n}\right): \sum_{n} s_{n}=s\right\} .
$$

(c) The absolutely convex hull of $A$ denoted by $\kappa(A)$ is norm dense in the unit ball of $S(A)$. Thus $[A]$, the span of $A$, is dense in $S(A)$.

(d) Every $B K$-space $S$ is of the form $S(A)$ where $A$ is a balanced subset of $\omega$.

Proof. (a) Since $A$ is bounded in $\omega$,

$$
a_{j}=\sup \{|s(j)|: s \in A\}<\infty
$$

for each $j$. Thus if $\sum_{n} p\left(s_{n}\right)<\infty$

$$
\sum_{n}\left|s_{n}(j)\right| \leqq \sum_{n} a_{j} p\left(s_{n}\right)<\infty \text {. }
$$

(b) The routine proof that \|\|$^{A}$ is a seminorm and that $S(A)$ is a linear space is omitted. 
If $\|s\|^{A}=0$ then for each $\varepsilon>0$ there is a sequence $\left\{s_{n}\right\}$ in $\omega$ such that $\sum_{n} s_{n}=s$ and $\sum_{n} p\left(s_{n}\right)<\varepsilon$. Thus if $a_{j}$ is given by (4.2)

$$
|s(j)| \leqq a_{j} \varepsilon
$$

for each $j$. This implies that \|\|$^{A}$ is a norm since $s(j)=0$ for each $j$. For $s \in S(A)$ and each $j$

$$
\left|E_{j}(s)\right| \leqq a_{j} \sum_{n} p\left(s_{n}\right)
$$

whenever $\sum_{n} s_{n}=s$. Thus

$$
\left|E_{j}(s)\right| \leqq a_{j}\|s\|^{A}
$$

so that $S(A)$ is a normed $K$-space.

Let $\left\{s_{n}\right\}$ be a sequence in $S(A)$ such that $\sum_{n}\left\|s_{n}\right\|^{A}<\infty$. For each $n$ let

$$
s_{n}=\sum_{j=1}^{\infty} s_{n j}, \sum_{j=1}^{\infty} p\left(s_{n j}\right)<\left\|s_{n}\right\|^{A}+\varepsilon / 2 n,
$$

and let

$$
s=\sum_{n=1}^{\infty} \sum_{j=1}^{\infty} s_{n j} .
$$

Then $s$ is in $S(A)$ and $\left\{\sum_{n=1}^{k} s_{n}\right\}$ converges to $s$ since

$$
\begin{aligned}
\left\|s-\sum_{n=1}^{k} s_{n}\right\|^{A} & \leqq \sum_{n=k+1}^{\infty} \sum_{j=1}^{\infty} p\left(s_{n j}\right) \\
& \leqq \varepsilon / 2^{k}+\sum_{n=k+1}^{\infty}\left\|s_{n}\right\|^{A} .
\end{aligned}
$$

Therefore, $S(A)$ is a complete space.

(c) If $\|s\|^{A}<1$ there is a sequence $\left\{s_{n}\right\} \subset A$ such that

$$
s=\sum_{n=1}^{\infty} s_{n} \quad \text { and } \quad \sum_{n=1}^{\infty} p\left(s_{n}\right)<1 .
$$

Since $A$ is balanced $\sum_{n=1}^{k} s_{n}$ is in $\kappa(A)$ for each $k$ and

$$
\lim _{k}\left\|\sum_{n=1}^{k} s_{n}-s\right\|^{A}=0 \text {. }
$$

Thus $\kappa(A)$ is dense in the unit ball of $S(A)$.

(d) Trivial. Let $A$ be the unit ball of $S$.

Let $A$ and $B$ be two subsets of $\omega$. If there is a number $a>0$ such that $A \subset a B$ then $B$ is said to absorb $A$. If $A$ absorbs $B$ and $B$ absorbs $A$ then $A$ and $B$ are called equivalent. If $p$ is the Minkowski gauge of a balanced set $A$ and \|\|$^{A}$ the norm given by (4.1) then the set

$$
B=\left\{s \in[A]:\|s\|^{A} \leqq 1\right\}
$$


contains $\kappa(A)$, but $\kappa(A)$ may not absorb $B$. For example, let $t$ denote the sequence $(1,1 / 2,1 / 4, \cdots)$ and define $p$ on $\varphi \oplus[\{t\}]$ by

$$
p(s+a t)=\sum_{j}|s(j)|+|a| .
$$

Then $2^{n}\left(t-\sum_{j=1}^{n+1} t(j) e_{j}\right)$ is in $B$ for each $n$ whereas

$$
p\left(2^{n}\left(t-\sum_{j=1}^{n+1} t(j) e_{j}\right)\right)>2^{n} .
$$

4.2. Let $A$ be a bounded absolutely convex subset of $\omega$; let $T=[A]$; let $p$ be the Minkowski gauge of $A$; and let \|\|$^{A}$ be given by (4.1). Then the following statements are equivalent

(a) If $\sum_{n=1}^{\infty} s_{n}=s$ coordinatewise then $p(s) \leqq \sum_{n=1}^{\infty} p\left(s_{n}\right)$.

(b) There exists $K$ such that $p(s) \leqq K \sum_{n=1}^{\infty} p\left(s_{n}\right)$ whenever $\sum_{n=1}^{\infty} s_{n}=s$ coordinatewise.

(c) There is $K$ such that $p(s) \leqq K\|s\|^{A}$ for $s$ in $T$.

(d) There is a $B K$-space $S$ of which the normed space $(T, p)$ is a topological subspace.

(e) If $\hat{E}_{j}$ denotes the extension of $E_{j}$ to the completion $\hat{T}$ of $(T, p)$ then $\left\{\hat{E}_{j}\right\}$ is total on $\widehat{T}$.

(f) If $\left\{s_{n}: n=1,2, \cdots\right\}$ is a Cauchy sequence in $(T, p)$ such that $s_{n}(j) \rightarrow 0$ for each $j$ then $\lim _{n} p\left(s_{n}\right)=0$.

(g) $p(s)=\|s\|^{A}$ for $s$ in $T$.

Proof. First note that $(T, p)$ is a $K$-space since $A$ is bounded in $\omega$.

$(\mathrm{a}) \Rightarrow(\mathrm{b}) \Rightarrow(\mathrm{c})$. Obvious.

(c) $\Rightarrow$ (d). Let $S=S(A)$. By definition $\|s\|^{A} \leqq p(s)$ for $s$ in $T$ so that (c) implies $(T, p)$ is a topological subspace of $S(A)$.

$(\mathrm{d}) \Rightarrow(\mathrm{e})$. By 4.1(c), $T$ is dense in $S(A)$, and if (d) holds, $(T, P)$ is a topological subspace of $S(A)$. Thus $S(A)$ is isomorphic to $\hat{T}$ and the set of functionals $\left\{E_{j}\right\}$ defined on $S(A)$ as total.

(e) $\Rightarrow(\mathrm{f})$. Let $x=\lim _{n} s_{n}$ in $\hat{T}$. If $\left\{E_{j}\right\}$ is total on $\hat{T}$ then $x=0$ since $\hat{E}_{j}(x)=\lim _{n} E_{j}\left(s_{n}\right)=0$ for each $j$. Thus $\lim _{n} p\left(s_{n}\right)=p(x)=0$.

(f) $\Rightarrow$ (a). It may be assumed that $\sum_{n=1}^{\infty} p\left(s_{n}\right)<\infty$. Then

$$
\left\{\sum_{n=k}^{\infty} s_{n}: k=1,2, \cdots\right\}
$$

is a Cauchy sequence in $(T, p)$ such that $\sum_{n=k}^{\infty} s_{n}(j) \rightarrow 0$ for each $j$. Thus $\lim _{k} p\left(\sum_{n=k}^{\infty} s_{n}\right)=0$ by (f) so that (a) holds by passing to the limit in the inequality

$$
p(s) \leqq \sum_{n=1}^{k} p\left(s_{n}\right)+p\left(\sum_{n=k+1}^{\infty} s_{n}\right) .
$$

$(\mathrm{a}) \Rightarrow(\mathrm{g}) \Rightarrow(\mathrm{c})$. Obvious. 
Definition 4.3. A bounded absolutely convex subset $A$ of $\omega$ will be called consistent if one, hence all, of the conditions of 4.2 is satisfied. A bounded subset $A$ of $\omega$ which is balanced will be called consistent if $\kappa(A)$ is consistent.

See 3.1 of [10] in connection with the idea of consistency.

\section{Representation.}

THEOREM 5.1. (a) A sequence space is associated with a complete biorthogonal sequence in a Banach space if and only if it is of the form $S(A)$ where $A$ is a balanced subset of $\varphi$ which absorbs each point of $\varphi$.

(b) In addition it may be assumed that $A^{(\omega)(\varphi)}=A$ and $A$ is consistent.

(c) The complete biorthogonal sequence is norming if and only if the associated sequence space is of the form $S(A)$ where $A^{(\varphi)(\varphi)}=A$.

Proof. (a) If $A$ is a subset of $\varphi$ having the properties listed in (a) then by $4.1, S(A)$ is a $B K$-space in which $\left\{e_{j}, E_{j}\right\}$ is a complete biorthogonal sequence.

If $S$ is a sequence space associated with a complete biorthogonal sequence in a Banach space let $A$ consist of all $s \in \varphi$ such that $\|s\| \leqq 1$ where \|\| is the identity norm on $S$ obtained in $\S 3$. If $p$ is the Minkowski gauge of $A$ then $p(s)=\|s\|$ for $s$ in $\varphi$ so that $A$ is consistent by 4.2. Since $\varphi$ is dense in $S$ and in $S(A)$ the spaces are equal.

(b) It has already been noted that the set $A$ defined in the previous paragraph is consistent. Furthermore $A$ is absolutely convex and closed in the relative topology of $S$ on $\varphi$ so that $A^{(\omega)(\varphi)}=A$.

(c) If $S$ has the form $S(A)$ where $A=A^{(\varphi)(\varphi)}$ then for $s$ in $\varphi$

$$
p(s)=\sup \left\{|(s, t)|: t \in A^{(\varphi)}\right\} .
$$

Here $p$ is the Minkowski gauge of $A$. Thus by 4.2(a) it follows that $A$ is consistent and for $s \in S(A)$

$$
\|s\|^{A}=\sup \left\{|(s, t)|: t \in A^{(\varphi)}\right\}
$$

so that $\left\{e_{j}, E_{j}\right\}$ is a norming complete biorthogonal sequence in $S(A)$.

If $S$ is associated with a complete norming biorthogonal sequence in a Banach space let $B$ be a subset of $\varphi$ such that the norm

$$
\|s\|=\sup \{|(s, t)|: t \in B\}
$$

determines the topology of $S$. If $A=B^{(\varphi)}$ then $S=S(A)$ and $A^{(\varphi)(\varphi)}=$ $B^{(\varphi)(\varphi)(\varphi)}=A$ because of the bipolar theorem. 
For $S$ a $B K$-space containing $\varphi, S^{o}$ denotes the closure of $\varphi$ in $S$ and $S^{f}$ denotes the space of all sequences of the form $\left\{x^{\prime}\left(e_{j}\right): j=1,2, \cdots\right\}$, where $x^{\prime} \in S^{*}$. Note that if $S=S^{o}$ then $S^{f}$ has the same meaning as in $\S 3$.

5.2. Let $S$ be a $B K$-space containing $\varphi$ and having norm \|\| , and let $A=\{s \in \varphi:\|s\| \leqq 1\}$.

(a) $S^{f}=S^{o f}$.

(b) With the norm

$$
\|t\|_{A}=\sup \{|(t, s)|: s \in A\}
$$

$S^{f}$ is a $B K$-space isometric to $S^{0^{*}}$ under the correspondence of $x^{\prime}$ in $S^{o^{*}}$ to $\left\{x^{\prime}\left(e_{j}\right)\right\}$ in $S^{f}$.

(c) $S^{f}=\bigcup_{n=1}^{\infty} n A^{(\omega)}$.

(d) $\left\{e_{j}, E_{j}\right\}$ is a norming complete biorthogonal sequence in $S^{f o}$.

Proof. (a) Since each continuous linear functional on $S$ is continuous and linear when restricted to $S^{o}, S^{f} \subset S^{o f}$. Since each continuous linear functional on $S^{o}$ can be extended to $S$ by the HahnBanach theorem $S^{o f} \subset S^{f}$.

(b) Since $\left\{e_{j}, E_{j}\right\}$ is a complete biorthogonal sequence in $S^{o}, S^{f}$ is a isometric to $S^{0^{*}}$ with the norm

$$
\begin{aligned}
\left\|t_{y}\right\|_{f} & =\sup \left\{|y(s)|: s \in S^{o} \text { and }\|s\| \leqq 1\right\} \\
& =\sup \{|y(s)|: y \in A\}
\end{aligned}
$$

since $A$ is dense in the unit ball of $S^{o}$. But for $s$ in $\varphi$

$$
y(s)=\sum_{j} s(j) y\left(e_{j}\right)=\left(s, t_{y}\right) \text {. }
$$

(c) Because of (b) the unit ball of $S^{f}$ is part of $A^{(\omega)}$ so that $S^{f} \subset \bigcup_{n=1}^{\infty} n A^{(\omega)}$.

On the other hand let $t \in n A^{(\omega)}$ for some $n$. Define the functional $x_{t}^{\prime}$ on $\varphi$ by

$$
x_{t}^{\prime}(s)=(s, t)
$$

Since $t \in n A^{(\omega)}$

$$
\left|x_{t}^{\prime}(s)\right| \leqq n\|s\|
$$

for $s \in \varphi$ so that $x_{t}^{\prime}$ can be continuously extended to $S^{o}$. Since $x_{t}^{\prime}\left(e_{j}\right)=t(j)$ for each $j, t \in S^{f}$.

(d) This follows from (b).

Since

$$
\left\{t \in \varphi:\|t\|_{A} \leqq 1\right\}=A^{(\omega)} \cap \varphi=A^{(\varphi)}
$$


5.2(c) can be reapplied to determine that

$$
\begin{aligned}
S^{f f} & =\bigcup_{n=1}^{\infty} n A^{(\varphi)(\omega)} \\
S^{f f f} & =\bigcup_{n=1}^{\infty} n A^{(\varphi)(\varphi)(\omega)} \\
S^{f f f f} & =\bigcup_{n=1}^{\infty} n A^{(\varphi)(\varphi)(\varphi)(\omega)}=S^{f f}
\end{aligned}
$$

since $A^{(\varphi)(\varphi)(\varphi)}=A^{(\varphi)}$ for a subset $A$ of $\varphi$ because of the bipolar theorem.

5.3. For $S$ a $B K$-space in which $\varphi$ is dense the following statements are equivalent:

(a) $\left\{e_{j}, E_{j}\right\}$ is a norming complete biorthogonal sequence in $S$.

(b) $S^{f f o}=S$.

(c) $S^{f f f}=S^{f}$.

Proof. $\quad(\mathrm{a}) \Rightarrow(\mathrm{b})$. If $\left\{e_{j}, E_{j}\right\}$ is a norming biorthogonal sequence there is a subset $A$ of $\varphi$ such that $A=A^{(\varphi)(\varphi)}$ and $S=S^{(A)}$. By 5.2(c) the norm \|\|$_{f f}$ on $S^{f f}$ is given by

$$
\|t\|_{f f}=\sup \left\{|(t, s)|: s \in A^{(\varphi)(\varphi)}\right\} \text {. }
$$

But since $A^{(\varphi)(\varphi)}=A,\|t\|_{f f}=\|t\|_{A}$ for $t \in S$. Thus $S=S^{f f o}$ since $\varphi$ is dense in both spaces.

$(b) \Rightarrow(c)$. If $S^{f f o}=S$ then their $B K$-topologies can be determined by the same norm \|\| . If $A=\{s \in \varphi$ : $\|s\| \leqq 1\}$ then both $S^{f f f}$ and $S^{f}$ are equal to $\bigcup_{n=1}^{\infty} n A^{(\omega)}$.

(c) $\Rightarrow$ (a). If $S^{f}=S^{f f f}$ then the absolutely convex sets $A^{(\omega)}$ and $A^{(\varphi)(\varphi)(\omega)}$ are equivalent since the first is the closed unit ball of $S^{f}$ and the second is the closed unit ball of $S^{f f f}$. Thus $A^{(\omega)(\varphi)}$ is equivalent to $A^{(\varphi)(\varphi)(\omega)(\varphi)}=A^{(\varphi)(\varphi)}$ and so $S=S(A)=S\left(A^{(\varphi)(\varphi)}\right)$ which implies that $\left\{e_{j}, E_{j}\right\}$ is norming in $S$ by 5.1 .

The following representation theorem is an immediate consequence of 5.2 and 5.3 .

THEOREM 5.4. Let $S$ be a sequence space associated with a complete biorthogonal sequence in a Banach space $X$. If $S$ is equal to $S(A)$ where $A$ is a consistent balanced subset of $\varphi$ then $X^{*}$ is isomorphic to $S^{f}=S\left(A^{(\omega)}\right)=\bigcup_{n=1}^{\infty} n A^{(\omega)}$. The given complete biorthogonal sequence is norming if and only if $S^{f f f}=S^{f}$.

6. The series space and series summable biorthogonal sequences. Let $S$ be a sequence space associated with a complete biorthogonal sequence in a Banach space and let $A$ consist of all $s$ in 
$\varphi$ with $\|s\| \leqq 1$. Since the Minkowski gauge of the balanced set $A A^{(\omega)}$ is

$$
p(v)=\inf \left\{\|s\|\|t\|_{f}: s \in \varphi, t \in S^{f} \text { and } s t=v\right\}
$$

$\mathscr{S}(S)$ as defined by (3.1) and (3.2) is the space $S\left(A A^{(\omega)}\right)$ in the sense of Proposition 4.1. The following statement thus follows immediately.

6.1. With the norm

$$
\|v\|=\inf \left\{\sum_{n=1}^{\infty}\left\|s_{n}\right\|\left\|t_{n}\right\|_{f}: \sum_{n=1}^{\infty} s_{n} t_{n}=v, s_{n} \in \varphi, t_{n} \in S^{f}\right\},
$$

$\mathscr{S}(S)$ is a $B K$-space in which $\varphi$ is dense.

Since $\varphi$ is dense in $S$ it can be shown that $\mathscr{S}(S)$ consists of all sequences $v$ having the form

$$
v=\sum_{j} s_{j} t_{j}, s_{j} \in S, t_{j} \in S^{f} ; \sum_{j}\left\|s_{j}\right\|\left\|t_{j}\right\|_{f}<\infty
$$

and that

$$
\|v\|_{S}=\inf \left\{\sum_{j}\left\|s_{j}\right\|^{A}\left\|t_{j}\right\|_{A}: \sum_{j} s_{j} t_{j}=v, s_{j} \in S, t_{j} \in S^{f}\right\} .
$$

For $X$ a Banach space let $\mathscr{N}(X)$ denote the Banach space of all nuclear operators $F$ from $X$ into $X$ with the norm

$$
\|F\|=\inf \left\{\sum_{j}\left\|y_{j}^{\prime}\right\|\left\|y_{j}\right\|: y_{j} \in X, y_{j}^{\prime} \in X^{*}, \sum_{j} y_{j}^{\prime}(x) y_{j}=F(x) \text { for } x \in X\right\} \text {. }
$$

See 3.1 .3 of [9].

6.2. $\mathscr{S}(S)$ consists of all sequences of the form

$$
v_{F}=\left\{E_{j}\left(F e_{j}\right)\right\}
$$

as $F$ ranges over $\mathscr{N}(S)$. The function defined by

$$
\Delta(F)=v_{F}
$$

is a continuous linear operator from $\mathscr{N}(S)$ onto $\mathscr{S}(S)$.

Proof. It is first shown that if $F \in \mathscr{N}(S)$ then $\Delta(F) \in \mathscr{S}(S)$. Suppose for each $s \in S$

$$
F(s)=\sum_{n} y_{n}(s) s_{n}, y_{n} \in S^{*}, s_{n} \in S, \sum_{n}\left\|y_{n}\right\|\left\|s_{n}\right\|<\infty .
$$

Let $t_{n}$ in $S^{f}$ be such that $t_{n}(j)=y_{n}\left(e_{j}\right)$ for each $n$ and each $j$. Then for each $j$

$$
\sum_{n} s_{n}(j) t_{n}(j)=\sum_{n} E_{j}\left(s_{n}\right) y_{n}\left(e_{j}\right)=E_{j}\left(F e_{j}\right)=v_{F}(j)
$$


so that $\sum_{n} s_{n} t_{n}=v_{F}$. Since $\sum_{n}\left\|s_{n}\right\|\left\|t_{n}\right\|_{f}<\infty, v_{F} \in \mathscr{S}(S)$.

It is clear that $\Delta$ is linear. Since $F(x)=\sum_{n} y_{n}^{\prime}(x) y_{n}$ implies that $E_{j}\left(F e_{j}\right)=\sum_{n} y_{n}^{\prime}\left(e_{j}\right) E_{j}\left(y_{n}\right)$ for each $j,\left\|v_{F}\right\|, \leqq\|F\|$ because the first norm is defined as an infimum over a larger set. Therefore, $\Delta$ is continuous.

$$
\text { 6.3. } \mathscr{S}(S)^{f} \subset M(S) \subset M(\mathscr{S}(S)) \text {. }
$$

Proof. $\mathscr{S}(S)^{f} \subset M(S)$. Let $B$ denote the set of all $v \in \varphi$ such that $\|v\|_{\mathscr{S}} \leqq 1$ and let $A$ be the set of all $s \in \varphi$ such that $\|s\| \leqq 1$. Then $A A^{(\omega)} \subset B$ so that

$$
\begin{gathered}
\mathscr{S}(S)^{f}=\bigcup_{n} n B^{(\omega)} \subset \bigcup_{n} n\left(A A^{(\omega)}\right)^{(\omega)}=M(S) . \\
M(S) \subset M(\mathscr{S}(S)) \text {. If } u \in M(S) \text { and } v \in \mathscr{S}(S) \text {, let } v=\sum_{n} s_{n} t_{n} .
\end{gathered}
$$
Then $u v=\sum_{n} u s_{n} t_{n}$ and since

$$
\sum_{n}\left\|u s_{n}\right\|\left\|t_{n}\right\|_{f} \leqq \sum_{n}\|u\|_{M}\left\|s_{n}\right\|\left\|t_{n}\right\|_{f}<\infty
$$

it follows that $u v \in \mathscr{S}(S)$. Thus $u \in M(\mathscr{S}(S))$.

From equation (6.1) it follows that $S S^{f} \subset \mathscr{S}(S)$.

THEOREM 6.4. Let $S$ be a sequence space associated with a complete biorthogonal sequence in a Banach space and let $A$ be a consistent absolutely convex subset of $\varphi$ such that \|\|$^{A}$ determines the BKtopology on $S$. Then the following statements are equivalent:

(a) $A A^{(\omega)}$ is a consistent subset of $\varphi$;

(b) $\mathscr{S}(S)^{f}=M(S)$;

(c) $e \in \mathscr{S}(S)^{f}$.

(d) There is a continuous linear functional $E$ on $\mathscr{S}(S)$ such that if $s_{x} \in S$ and $t_{x}^{\prime} \in S^{f}$ then

$$
E(s t)=x^{\prime}(x) .
$$

Proof. $\quad(a) \Rightarrow(b)$. If $A A^{(\omega)}$ is consistent then by $5.2(\mathrm{c})$

$$
\mathscr{S}(S)^{f}=S\left(A A^{(\omega)}\right)^{f}=\bigcup_{n} n\left(A A^{(\omega)}\right)^{(\omega)}=M(S) .
$$

$(\mathrm{b}) \Rightarrow$ (c). $\quad e \in M(S)$.

$\left(\right.$ c) $\Rightarrow\left(\right.$ d). If $e \in \mathscr{P}(S)^{f}$ there is a continuous linear functional $E$ on $\mathscr{S}(S)$ such that $E\left(e_{j}\right)=1$ for each $j$.

Given $t \in S^{f}$ define $F_{t}$ from $S$ into $\mathscr{S}(S)$ by $F_{t} s=s t$. Then $F_{t}$ is a closed, hence a continuous operator. Thus the functional defined by

$$
y^{\prime}(s)=E\left(F_{t} s\right)=E(s t)
$$

is continuous linear functional on $S$. For each $s$ in $\varphi$ 


$$
y^{\prime}(s)=E(s t)=\sum_{j} s(j) t(j)=x_{t}^{\prime}(s)
$$

so that

$$
y^{\prime}(s)=E(s t)=x_{t}^{\prime}(s)
$$

for each $s$ in $S$ because $\phi$ is dense in $S$.

$(d) \Rightarrow$ (c). If $E$ is given by (d) then $E\left(e_{j}\right)=1$ for each $j$.

$(\mathrm{c}) \Rightarrow(\mathrm{b})$. If $e \in \mathscr{S}(S)^{f}$ then $M\left(\mathscr{S}(S)^{f}\right) \subset \mathscr{S}(S)^{f}$ for if $u \in M\left(\mathscr{S}(S)^{f}\right)$ then $u e=u \in \mathscr{S}(S)^{f}$. By $\quad 6.3 \quad \mathscr{S}(S)^{f} \subset M(S)$ and by 3.5 of [8] $M(T) \subset M\left(T^{f}\right)$ for every $B K$-space. Thus $\mathscr{S}(S)^{f}=M(S)$.

(b) $\Rightarrow$ (a). Let $B$ consist of all $v$ in $\varphi$ such that $\|v\|_{\infty} \leqq 1$. If $\mathscr{S}(S)^{f}=M(S)$ then $M(S)=\mathrm{U}_{n} n B^{(\omega)}$ by $5.2(\mathrm{c})$ and $B^{(\omega)}$ is a bounded barrel in $M(S)$. Thus $B^{(\omega)}$ is absorbed by $\left(A A^{(\omega)}\right)^{(\omega)}$ which implies that $\left(A A^{(\omega)}\right)^{(\omega)(\varphi)}$ absorbs $B$. Thus $\left(A A^{(\omega)}\right)$ is consistent.

A complete biorthogonal sequence in a Banach space will be called series summable if the associated sequence space has one, hence all four of the properties mentioned in 6.4.

6.5. Let $\left\{x_{j}, x_{j}^{\prime}\right\}$ be a series summable complete biorthogonal sequence in a Banach space $X$.

(a) If $x \in X$ and $x^{\prime} \in X^{*}$ then

$$
\sum_{j} x^{\prime}\left(x_{j}\right) x_{j}^{\prime}(x)=x^{\prime}(x)
$$

whenever the sum has finitely many nonzero terms.

(b) If $I_{1}$ and $I_{2}$ are complementary sets of indices then

$$
\operatorname{cl}\left[\left\{x_{j}: j \in I_{1}\right\}\right]=\cap\left\{x \in X: x_{\jmath}^{\prime}(x)=0, j \in I_{2}\right\}
$$

(cl means closure).

Proof. (a) Without loss of generality assume the biorthogonal sequence in $\left\{e_{j}, E_{j}\right\}$ in a $B K$-space $S$. Then

$$
x^{\prime}(s)=E\left(s t_{x^{\prime}}\right)=\sum_{j} s(j) t(j)
$$

since $s t \in \varphi$.

(b) If $j \in I_{1}$ and $k \in I_{2}$ then $x_{t}^{\prime}\left(x_{j}\right)=0$ so that the set on the left hand side of (4.6) is contained in that on the right.

If $x^{\prime}$ in $X^{*}$ is such that $x^{\prime}\left(x_{\jmath}\right)=0$ for $j$ in $I_{1}$ and $x$ is in the set on the right hand side of (4.6)

$$
x^{\prime}(x)=\sum_{j} x^{\prime}\left(x_{j}\right) x_{j}^{\prime}(x)=0
$$

by (a) since each summand is 0 . Thus $\left.\left[x_{j}: j \in I_{1}\right\}\right]$ is dense in the set on the right. 
Let $Z$ denote the infinite matrix whose element in the $i$ th row $j$ th column is given by

$$
a_{i j}=\left\{\begin{array}{lll}
1 & i=j, j+1 & j \text { odd } \\
1 / 2^{j} & i \geqq j & j \text { even } \\
0 & \text { otherwise. } &
\end{array}\right.
$$

Let $(Z)$ denote the space of all sequences $\left(z_{i}\right)$ such that $\lim _{n} \sum_{j=1}^{\infty} a_{i j} z_{j}$ exists. On p. 657 of [13] it is shown that $\left\{e_{j}, E_{j}\right\}$ is a complete biorthogonal sequence. But, as A. K. Snyder has noted, (6.3) does not hold since $\cap\left\{s \in(Z): E_{2 j}(s)=0\right\}$ is $c$. Thus $\left\{e_{j}, E_{j}\right\}$ is not series summable.

6.6. Every Banach space in which there is a series summable complete biorthogonal sequence has the approximation property (p. 167 of [5]).

Proof. Without loss of generality it may be assumed that the space is a $B K$-space $S$ in which $\left\{e_{j}, E_{j}\right\}$ is a series summable complete biorthogonal sequence. Suppose $\sum_{n}\left\|s_{n}\right\|\left\|y_{n}\right\|<\infty, s_{n} \in S, y_{n} \in S^{*}$ and $\sum_{n} y_{n}(s) s_{n}=0$ for each $s$ in $S$. Then $\sum_{n} y_{n}\left(e_{j}\right) s_{n}=0$ for each $j$ so that

$$
\sum_{n} y_{n}\left(s_{n}\right)=\sum_{n} E\left(s_{n} t_{y_{n}}\right)=E\left(\sum_{n} s_{n} t_{y_{n}}\right)=E(0)=0 .
$$

Thus the trace of a nuclear operator is well defined on $S$ so that by Proposition 35 of [5] $S$ has the approximation property.

Strongly series summable complete biorthogonal sequences.

7.1. Let $S$ be a sequence space associated with a complete biorthogonal sequence in a Banach space and let $A$ be an absolutely convex consistent subset of $\varphi$ such that \|\|$^{A}$ determines the topology of $S$ (i.e., $S=S(A)$ ).

(a) $M(S)=\bigcup_{n=1}^{\infty} n\left(A A^{(\omega)}\right)^{(\omega)}$.

(b) $\left\{e_{j}, E_{j}\right\}$ is a norming complete biorthogonal sequence in $M(S)^{0}$.

(c) $M(S)^{f f} \subset M(S)$.

Proof. (a ) As noted in $\S 3, M(S)$ is a $B K$-algebra with the norm

$$
\|u\|_{M}=\sup \left\{\|u s\|^{A}:\|s\|^{A} \leqq 1\right\} \text {. }
$$

But $A$ is dense in the unit ball of $S$ so that

$$
\begin{aligned}
\|u\|_{M} & =\sup \left\{\|u s\|^{A}: s \in A\right\} \\
& =\sup \left\{|(u s, t)|: s \in A, t \in A^{(\omega)}\right\} \\
& =\sup \left\{|(u, v)|: v \in A A^{(\omega)}\right\} .
\end{aligned}
$$


Hence

$$
M(S) \subset \bigcup_{n=1}^{\infty} n\left(A A^{(\omega)}\right)^{(\omega)}
$$

If $u \in n\left(A A^{(\omega)}\right)^{(\omega)}$ for some $n$, define $F_{u}^{\prime}$ on $\varphi$ by

$$
F_{u}^{\prime}(s)=u s \text {. }
$$

Then $F_{u}^{\prime \prime}$ is continuous on $\left(\varphi,\|\|^{A}\right)$ since

$$
\|u s\|^{A}=\sup \left\{|(u s, t)|: t \in A^{(\omega)}\right\} \leqq n\|s\|^{A}
$$

for $s$ in $\varphi$. Thus $F_{u}^{\prime \prime}$ can be extended continuously to all of $S$, and if $F_{u}$ is this extension

$$
F_{u} s=u s \text {. }
$$

Thus $u \in M(S)$ so that equality holds in (7.1).

(b) This follows from the equality $\|u\|_{M}=\sup \left\{|(u, v)|: v \in A A^{(\omega)}\right\}$.

(c) By 5.2(c)

$$
M(S)^{f f}=\bigcup_{n=1}^{\infty} n\left(A A^{(\omega)}\right)^{(\varphi)(\varphi)(\omega)}
$$

which is contained in $M(S)$ since

$$
\left(A A^{(\omega)}\right)^{(\varphi)(\varphi)(\omega)} \subset\left(A A^{(\omega)}\right)^{(\omega)} .
$$

Since $A A^{(\omega)} \subset\left(A A^{(\omega)}\right)^{(\varphi)(\varphi)}$ it follows that

$$
S S^{f} \subset \mathscr{S}(S) \subset M(S)^{f o} .
$$

THEOREM 7.2. Let $S$ be a sequence space associated with a complete biorthogonal sequence in a Banach space, and let $A$ be a consistent absolutely convex subset of $\phi$ such that \|\|$^{A}$ determines the topology of $S$. Then the following statements are equivalent

(a) $M(S)^{f f}=M(S)$

(b) $e \in M(S)^{f f}$

(c) There is a sequence $\left\{u_{n}\right\}$ in $\varphi$ such that $\lim _{n} u_{n}(j)=1$ for each $j$ and $\left\{u_{n}\right\}$ is bounded in $M(S)$.

(d) There is a sequence $\left\{u_{n}\right\}$ in $\varphi$ such that

$$
\lim _{n}\left\|s-u_{n} s\right\|^{A}=0
$$

for each $s \in S$.

(e) There is a sequence $\left\{u_{n}\right\}$ in $\varnothing$ such that $\lim _{n}\left(s u_{n}, t_{y}\right)=y(s)$ for $s$ in $S$ and $y \in S^{*}$.

(f) $\left(A A^{(\omega)}\right)^{(\varphi)(\varphi)(\omega)}$ absorbs $\left(A A^{(\omega)}\right)^{(\omega)}$.

Proof. (a) $\Rightarrow(\mathrm{b})$. Trivial since $e \in M(S)$.

(b) $\Rightarrow$ (c). If $e \in M(S)^{f f}$ then $e$ is in $a\left(A A^{(\omega)}\right)^{(\varphi)(\varphi)(\omega)}$ for some $a>0$ 
because $\left(A A^{(\omega)}\right)^{(\varphi)(\varphi)(\omega)}$ is the unit ball of $M(S)^{f f}$ by $5.2(\mathrm{c})$. There is thus a sequence $\left\{u_{n}\right\}$ in $\left(A A^{(\omega)}\right)^{(\varphi)}$ converging coordinatewise to $e$ since $\left(A A^{(\omega)}\right)^{(\varphi)(\varphi)(\omega)}$ is the closure in $\omega$ of $\left(A A^{(\omega)}\right)^{(\varphi)}$. Also each $u_{n}$ is in the unit ball of $M(S)$.

$(\mathrm{c}) \Rightarrow(\mathrm{d})$. Let $\left\{u_{n}\right\}$ be given by (c). For $s$ in $\varphi$

$$
\lim _{n}\left\|s u_{n}-s\right\|^{A}=0
$$

since

$$
\left\|s-s u_{n}\right\|^{A} \leqq \sum_{j=1}^{N_{s}}\left|s(j)-s(j) u_{n}(j)\right|\left\|e_{j}\right\|^{A}
$$

where $N_{s}$ is such that $s(j)=0$ for $j>N_{s}$. Also

$$
\left\|s u_{n}\right\|^{A} \leqq\left\|u_{n}\right\|_{M}\|s\|^{A}
$$

for $s \in S$. Thus by the Banach-Steinhaus theorem (7.1) holds for each $s$ in $S$.

$(\mathrm{d}) \Rightarrow(\mathrm{e})$. Let $\left\{u_{n}\right\}$ be given by (d) then for $s$ in $S$ and $y$ in $S^{*}$

$$
\lim _{n}\left(s u_{n}, t_{y}\right)=y\left(\lim _{n} s u_{n}\right)=y(s) \text {. }
$$

$(\mathrm{e}) \Rightarrow(\mathrm{d})$. Let $\left\{u_{n}\right\}$ be given by (e) then (d) follows from II. 3.2 and II. 3.6 in [2] since $\lim _{n} u_{n} e_{k}=e_{k}$ for each $k$.

$(\mathrm{d}) \Longrightarrow(\mathrm{f})$. Let $\left\{u_{n}\right\}$ be given by (d). Given $u$ in $M(S)$ define $F_{u u_{n}}$ on $S$ by

$$
F_{u u_{n}} s=u u_{n} s \quad n=1,2, \cdots
$$

Then

$$
\lim _{n} F_{u u_{n}} s=u s
$$

for each $s$ in $S$. By the uniform boundedness principle $\left\{F_{u u_{n}}\right\}$ is a bounded sequence in $\mathscr{D}$. Thus

$$
\left\{u u_{n}\right\} \subset a\left(A A^{(\omega)}\right)^{(\omega)} \cap \varphi=a\left(A A^{(\omega)}\right)^{(\varphi)}
$$

for some $a>0$. Since $u_{n} e_{j} \rightarrow e_{j}$ for each $j,\left\{u u_{n}\right\}$ converges coordinatewise to $u$. Thus $u \in a\left(A A^{(\omega)}\right)^{(\varphi)(\varphi)(\omega)}$. This implies that $\left(A A^{(\omega)}\right)^{(\varphi)(\varphi)(\omega)}$ absorbs each point in $M(S)$. But $\left(A A^{(\omega)}\right)^{(\varphi)(\varphi)(\omega)}$ is closed in $M(S)$ since it is closed in $\omega$ and $M(S)$ is a $B K$-space. Thus $\left(A A^{(\omega)}\right)^{(\varphi)(\varphi)(\omega)}$ absorbs $\left(A A^{(\omega)}\right)^{(\omega)}$ which is the unit ball of $M(S)$.

(f) $\Rightarrow$ (a). By 7.1(c), $M(S)^{f f} \subset M(S)$. But $\left(A A^{(\omega)}\right)^{(\varphi)(\varphi)(\omega)}$ is the unit ball of $M(S)^{f f}$ so if it absorbs $\left(A A^{(\omega)}\right)^{(\omega)}, M(S) \subset M(S)^{f f}$.

A complete biorthogonal sequence in a Banach space is called strongly series summable if the associated sequence space has one, hence all of the properties in 7.2. 
7.3. Let $\left\{x_{j}, x_{j}^{\prime}\right\}$ be a strongly series summable complete biorthogonal sequence in a Banach space $X$. Then

(a) $\left\{x_{j}, x_{j}^{\prime}\right\}$ is norming;

(b) $\left\{x_{j}, x_{j}^{\prime}\right\}$ is a series summable basis;

(c) $\left\{x_{j}\right\}$ is an approximative basis in the sense of Singer ([11], Definition 3.19).

(d) $X$ has the metric approximation property (Definition 10, $\mathrm{p}$. 178 of [5]).

Proof. (a) Let $\left\{u_{n}\right\}$ be a sequence as in 7.2(d) and let

$$
B=\left\{\sum_{j} u_{n}(j) x^{\prime}\left(x_{j}\right) x_{j}^{\prime}: n=1,2, \cdots,\left\|x^{\prime}\right\| \leqq 1\right\} .
$$

It follows from $7.2(\mathrm{~d})$ that \|\|$_{B}$ determines the topology of $X$.

(b) By 7.1(a) and 5.2(c) the closed unit ball of $M(S)^{f}$ is $\left(A A^{(\omega)}\right)^{(\varphi)(\omega)}$ where $A=\{s \in \varphi:\|s\| \leqq 1\}$. This implies that $\mathscr{S}(S) \subset M(S)^{f}$ and since the inclusion is continuous, $\mathscr{S}(S) \subset M(S)^{f o}$. Thus if $M(S)^{f f}=M(S)$, $\mathscr{S}(S)^{f} \supset M(S)$ so that $\mathscr{S}(S)^{f}=M(S)$ by 6.3 .

(c) Let $y_{n j}^{\prime}$ be defined by

$$
y_{n j}^{\prime}(x)=u_{n}(j) x_{j}^{\prime}(x)
$$

$x \in X$.

Let $\left\{m_{n}\right\}$ be any increasing sequence of integers such that $u_{n}(j)=0$ for $j>m_{n}$. Then 7.2(d) implies that

$$
\lim _{n}\left\|x-\sum_{j=1}^{m_{n}} y_{n j}^{\prime}(x) x_{j}\right\|=0
$$

so that $\left\{x_{n}\right\}$ is an approximative basis of $X$.

(d) This is an immediate consequence of 7.2(d) with $F_{n}=F_{u_{n}}$ for each $n$.

TheOREM 7.4. Let $\left\{x_{j}, x_{j}^{\prime}\right\}$ and $\left\{y_{j}, y_{j}^{\prime}\right\}$ be complete biorthogonal sequences in Banach spaces. If $\left\{x_{j}, x_{j}^{\prime}\right\}$ is strongly series summable and $M\left(\left\{x_{j}\right\}\right) \subset M\left(\left\{y_{j}\right\}\right)$ then $\left\{y_{j}, y_{j}^{\prime}\right\}$ is a strongly series summable.

Proof. Let $S$ be the sequence space associated with $\left\{x_{j}, x_{j}^{\prime}\right\}$ and $T$ the sequence space associated with $\left\{y_{j}, y_{j}^{\prime}\right\}$. If $M(S) \subset M(T)$ the inclusion is continuous since both are $B K$-spaces. Thus if (c) holds for $M(S)$ it also holds for $M(T)$ with the same sequence $\left\{u_{n}\right\}$.

I do not know an example of a series summable complete biorthogonal sequence which is not strongly series summable. If $\left\{x_{j}, x_{j}^{\prime}\right\}$ is series summable and $\left\{e_{j}, E_{j}\right\}$ is norming in $\mathscr{S}(S)$ then $\mathscr{S}(S)=M(S)^{f o}$ so that $\left\{x_{j}, x_{j}^{\prime}\right\}$ is strongly series summable. 


\section{REFERENCES}

1. S. Banach, Théorie des opérations linéaires, Warsaw 1932.

2. N. Dunford and J. T. Schwartz, Linear operators, Part I, New York, 1958.

3. O. Frink Jr., Series expansions in linear vector spaces, Amer. J. Math. 63 (1941), 87-100.

4. V. F. Gaposhkin and M. I. Kadets, Operator bases in Banach spaces (Russian), Mat, Sb. (N. S.) (103) 61 (1963), p. 3-12.

5. A. Grothendieck, Produits tensoriels topologiques et espaces nucléaires, Memoirs Amer. Math. Soc. 16 (1955).

6. M. I. Kadets and A. Pełczynski, Basic sequences, biorthogonal systems and norming sets in Banach and Frechet spaces (Russian) Studia Math. 25 (1965), 297-323.

7. A. Markushevitch, Sur les bases (au sens large) dans les espaces lineaires, C. R. (Doklody) Acad. Sci. URSS (N. S.) 41 (1943), 227-229.

8. R. J. McGiveney and W. Ruckle, Multiplier algebras of biorthogonal systems (to appear in Pacific J. Math.)

9. A. Pietsch, Nukleare lokalkonvexe Raume, Schreftenreihe der Inst. fur Math. Deutsch. Acad. Wiss., Reithe A, 1, Berlin 1965.

10. W. Ruckle, Lattices of sequence spaces, Duke Math. J. 35 (1968), 491-504.

11. I. Singer, Baze in spatii Banach II, Studii si. Cercetari Matematice 15 (1964), 157-208.

12. A. Wilansky, Functional analysis, New York, 1964.

13. - Summability: the inset, replaceable matrices, the basis in summability space, Duke Math. J. 19 (1952), 647-660.

Received June 17, 1969, and in revised form February 10, 1970.

Clemson University 


\title{
PACIFIC JOURNAL OF MATHEMATICS
}

\author{
EDITORS
}

\author{
H. SAMELSON \\ Stanford University \\ Stanford, California 94305 \\ RichaRd PIERCE \\ University of Washington \\ Seattle, Washington 98105
}

J. DugundJI

Department of Mathematics

University of Southern California

Los Angeles, California 9.0007

RICHARD ARENS

University of California

Los Angeles, California 9.0024

\section{ASSOCIATE EDITORS}

E. F. BeCKenBACH
B. H. NeumanN

F. WoLE

K. YoSHIDA

\section{SUPPORTING INSTITUTIONS}

\author{
UNIVERSITY OF BRITISH COLUMBIA \\ CALIFORNIA INSTITUTE OF TECHNOLOGY \\ UNIVERSITY OF CALIFORNIA \\ MONTANA STATE UNIVERSITY \\ UNIVERSITY OF NEVADA \\ NEW MEXICO STATE UNIVERSITY \\ OREGON STATE UNIVERSITY \\ UNIVERSITY OF OREGON \\ OSAKA UNIVERSITY \\ UNIVERSITY OF SOUTHERN CALIFORNIA
}

\author{
STANFORD UNIVERSITY \\ UNIVERSITY OF TOKYO \\ UNIVERSITY OF UTAH \\ WASHINGTON STATE UNIVERSITY \\ UNIVERSITY OF WASHINGTON \\ AMERICAN MATHEMATICAL SOCIETY \\ CHEVRON RESEARCH CORPORATION \\ TRW SYSTEMS \\ NAVAL WEAPONS CENTER
}

The Supporting Institutions listed above contribute to the cost of publication of this Journal, but they are not owners or publishers and have no responsibility for its content or policies.

Mathematical papers intended for publication in the Pacific Journal of Mathematics should be in typed form or offset-reproduced, (not dittoed), double spaced with large margins. Underline Greek letters in red, German in green, and script in blue. The first paragraph or two must be capable of being used separately as a synopsis of the entire paper. The editorial "we" must not be used in the synopsis, and items of the bibliography should not be cited there unless absolutely necessary, in which case they must be identified by author and Journal, rather than by item number. Manuscripts, in duplicate if possible, may be sent to any one of the four editors. Please classify according to the scheme of Math. Rev. Index to Vol. 39. All other communications to the editors should be addressed to the managing editor, Richard Arens, University of California, Los Angeles, California, 90024.

50 reprints are provided free for each article; additional copies may be obtained at cost in multiples of 50 .

The Pacific Journal of Mathematics is published monthly. Effective with Volume 16 the price per volume (3 numbers) is $\$ 8.00$; single issues, $\$ 3.00$. Special price for current issues to individual faculty members of supporting institutions and to individual members of the American Mathematical Society: $\$ 4.00$ per volume; single issues $\$ 1.50$. Back numbers are available.

Subscriptions, orders for back numbers, and changes of address should be sent to Pacific Journal of Mathematics, 103 Highland Boulevard, Berkeley, California, 94708.

PUBLISHED BY PACIFIC JOURNAL OF MATHEMATICS, A NON-PROFIT CORPORATION

Printed at Kokusai Bunken Insatsusha (International Academic Printing Co., Ltd.), 7-17, Fujimi 2-chome, Chiyoda-ku, Tokyo, Japan. 


\section{Pacific Journal of Mathematics}

\section{Vol. 34, No. $2 \quad$ June, 1970}

Shair Ahmad, On the oscillation of solutions of a class of linear fourth order

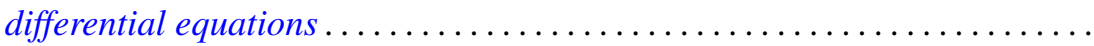

Leonard Asimow and Alan John Ellis, Facial decomposition of linearly

compact simplexes and separation of functions on cones ..............

Kirby Alan Baker and Albert Robert Stralka, Compact, distributive lattices of

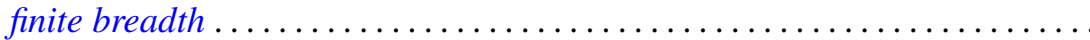

James W. Cannon, Sets which can be missed by side approximations to

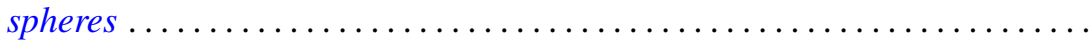

Prem Chandra, Absolute summability by Riesz means .................. 335

Francis T. Christoph, Free topological semigroups and embedding topological semigroups in topological groups....

Henry Bruce Cohen and Francis E. Sullivan, Projecting onto cycles in smooth, reflexive Banach spaces.................................

John Dauns, Power series semigroup rings .......................

Robert E. Dressler, A density which counts multiplicity ................

Kent Ralph Fuller, Primary rings and double centralizers ................

Gary Allen Gislason, On the existence question for a family of products.......

Alan Stuart Gleit, On the structure topology of simplex spaces .............

William R. Gordon and Marvin David Marcus, An analysis of equality in

certain matrix inequalities. $I \ldots \ldots \ldots \ldots \ldots \ldots \ldots \ldots$

Gerald William Johnson and David Lee Skoug, Operator-valued Feynman

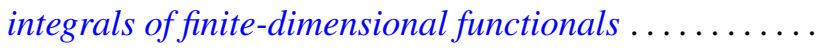

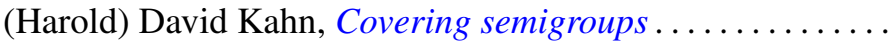

Keith Milo Kendig, Fibrations of analytic varieties

Norman Yeomans Luther, Weak denseness of nonatomic measures on perfect,

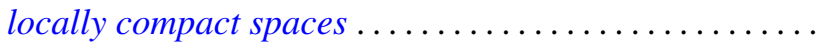

Guillermo Owen, The four-person constant-sum games; Discriminatory solutions on the main diagonal ...

Stephen Parrott, Unitary dilations for commuting contractions

Roy Martin Rakestraw, Extremal elements of the convex cone $A_{n}$ of

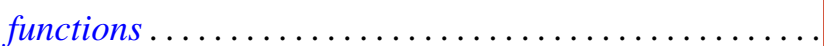

Peter Lewis Renz, Intersection representations of graphs by

William Henry Ruckle, Representation and series summability of complete

biorthogonal sequences.

F. Dennis Sentilles, The strict topology on bounded sets ...

Saharon Shelah, A note on Hanf numbers ...

Harold Simmons, The solution of a decision problem for several classes of rings. . .

Kenneth S. Williams, Finite transformation formulae involving the Legendre 